\title{
Clinical Reasoning: A 55-Year-Old Woman With Recurrent Episodes of Aphasia and Vision Changes
}

Author(s):

Valerie Jeanneret, MD ${ }^{1}$; Stewart Neill, MD² ${ }^{2}$ James G Greene, MD, PHD ${ }^{1}$; Olivia Groover, MD ${ }^{1}$; Carlos S Kase, $\mathrm{MD}^{1}$

\section{Corresponding Author:}

Valerie Jeanneret vjeanne@emory.edu

Neurology® Published Ahead of Print articles have been peer reviewed and accepted for publication. This manuscript will be published in its final form after copyediting, page composition, and review of proofs. Errors that could affect the content may be corrected during these processes. 
Affiliation Information for All Authors: 1. Emory University School of Medicine, Department of Neurology, Atlanta, Georgia; 2. Emory University School of Medicine, Pathology Department, Atlanta, Georgia

\section{Contributions:}

Valerie Jeanneret: Drafting/revision of the manuscript for content, including medical writing for content; Major role in the acquisition of data; Study concept or design

Stewart Neill: Drafting/revision of the manuscript for content, including medical writing for content James G Greene: Drafting/revision of the manuscript for content, including medical writing for content Olivia Groover: Drafting/revision of the manuscript for content, including medical writing for content Carlos S Kase: Drafting/revision of the manuscript for content, including medical writing for content

Number of characters in title: 73

Abstract Word count: 114

Word count of main text: 1500

References: 17

Figures: 2

Tables: 0

Supplemental: Patient consent form;Permission to use table; Supplemental Figure

Search Terms: [ 7 ] Intracerebral hemorrhage, [ 14 ] All Clinical Neurology, [ 132] Autoimmune diseases

Study Funding: The authors report no targeted funding

Disclosures: The authors report no disclosures relevant to the manuscript.

\section{Abstract}

A 55-year-old woman presented with recurrent episodes of headache, vision changes and language disturbances. Brain MRI showed multifocal white matter lesions, microhemorrhages, and enlarged perivascular spaces. After an extensive and unrevealing workup, she underwent a biopsy of brain and meninges that revealed thick and hyalinized leptomeningeal and cortical vessel walls that were strongly positive for ß-amyloid by immunohistochemical staining, suggestive of cerebral amyloid angiopathy (CAA). CAA can present as a spectrum of inflammatory responses to the deposition of amyloid- $ß$ in the vessel walls. Her clinical presentation, radiological and histopathological findings supported a diagnosis of probable 
CAA-related inflammation (CAA-ri). Although an uncommon entity, it is important to recognize it because most patients respond to immunosuppressive therapy.

\section{Case presentation}

A 55-year-old woman presented with acute onset of headache, inability to reach objects she could clearly see, vision changes (objects remaining in her vision after the stimulus was no longer present), and language disturbances. Her blood pressure ranged between 120/60 and 150/90. She was afebrile, with normal heart rate. Neurological exam was notable for difficulty naming and reading, with comprehension and repetition less affected, right homonymous hemianopsia. Her motor, sensory, and coordination functions were normal. Initial laboratory data were unremarkable, including HIV-negative status.

\section{Section 1}

1. What is the localization?

2. What is the differential diagnosis?

3. What tests would you obtain?

The visual phenomenon she described is known as palinopsia, a visual illusory phenomenon characterized by persistence of an image after the visual stimulus has been removed. It usually occurs with non-dominant parieto-occipital lesions and associates with a visual field defect, but can be seen with dominant or bilateral lesions, thought to be due to abnormal synthesis of visual information, occasionally related to focal seizures. ${ }^{1}$ Additionally, she described impairment of visually guided reaching, known as optic ataxia, typically associated with bilateral posterior parietal lesions. ${ }^{2}$ Given the visual field defect and motor predominant aphasia, in the absence of hemiparesis, we suspected a multifocal process, likely involving the left occipital or parieto-occipital and frontal regions.

Given the acute presentation, multifocal ischemic or hemorrhagic strokes were on the differential diagnosis. Inflammatory conditions were a possibility given the multifocal nature of her presentation.

She underwent brain magnetic resonance imaging (MRI) that revealed bilateral asymmetric 
cortical and subcortical T2/fluid-attenuated inversion recovery (FLAIR) hyperintense signal abnormalities, most prominent in the posterior regions (Figure 1, A), without enhancement, as well as cortical diffusion restriction of the left temporo-occipital region with features suggestive of vasogenic edema (Figure 1, B), and 2 punctate microhemorrhages within the right parietal lobe (Figure 1, C).

\section{Section 2}

\section{What is your differential diagnosis?}

2. What further steps would you take?

Imaging was suggestive of posterior reversible encephalopathy syndrome (PRES), which is associated with bilateral asymmetric T2/FLAIR hyperintensities in the subcortical white matter (WM) in the parieto-occipital lobes, occasionally associated with blood products. ${ }^{3}$ Although subcortical restricted diffusion can be seen in some cases, it is typically heterogeneous, with punctate lesions surrounded by vasogenic edema ${ }^{3}$. The cortical pattern of restricted diffusion raised the concern for seizures, ${ }^{4}$ which prompted continuous electroencephalogram (cEEG) demonstrating abundant left posterior temporal lateralized periodic discharges. She was started on levetiracetam, with improvement of her aphasia.

The lack of enhancement made inflammatory and neoplastic conditions less likely. Although extensive $\mathrm{WM}$ hyperintensities (WMH) without post-contrast enhancement raised the possibility of progressive multifocal leukoencephalopathy, this was considered unlikely given her immune-competent status and the absence of hypointense core with hyperintense rim on DWI sequence. ${ }^{5}$ PRES was highest in our differential diagnosis, and the lack of clear trigger led to extensive workup. Urine drug screen was negative. Cerebrospinal fluid (CSF) analysis revealed 1 WBC, 6 RBCs, protein 48 mg/dL (normal 15-45), and normal glucose. CSF cytology was negative for malignancy, and cultures were sterile. Extensive infectious workup was negative in the serum and CSF. No oligoclonal bands were detected. Her erythrocyte sedimentation rate $(51 \mathrm{~mm} / \mathrm{hr}$, normal <30) and C-reactive protein $(12.0 \mathrm{mg} / \mathrm{L}$, normal <10) were mildly elevated. She had a positive antinuclear antibody in a nucleolar pattern (titer 1:160) but no clinical evidence of lupus, scleroderma, or other connective tissue disease. Extractable 
nuclear antigen, double-stranded DNA, and antineutrophil cytoplasmic antibodies were negative, and complement levels were normal.

Although there were no clear triggers, except for mild hypertension, she was discharged with a presumed diagnosis of PRES. At 2-month follow-up, she had normal neurological exam, including language, attention, and memory. Shortly after, she was readmitted with similar episode of headache, visual and language difficulties. Repeat MRI (eFigure 1) showed new subcortical foci of FLAIR signal abnormalities with interval improvement of some of the previous lesions, asymmetric pachymeningeal and leptomeningeal enhancement, and new punctate microhemorrhage.

\section{Section 3}

\section{Does this change your differential diagnosis?}

2. What further testing would you obtain?

PRES can recur in 5-10\% of cases, however usually occurs with poorly controlled hypertension. ${ }^{3}$ Our patient was normotensive. Given recurrent headaches and focal neurological symptoms, we considered CNS vasculitis, although the presence of confluent areas of T2 hyperintensity without evidence of infarction would be unusual. ${ }^{6}$ Reversible cerebral vasoconstriction syndrome (RCVS) was considered as well. Similarities between PRES and RCVS have been described $^{7}$ and PRES-like reversible cerebral edema has been reported in $17-38 \%$ of patients with RCVS. ${ }^{8}$ She had a normal MRI angiogram on her initial presentation, as well as normal head CT angiogram and contrast angiogram this visit, making vasculopathy such as RCVS unlikely.

Autoimmune and inflammatory disorders such as recurrent ADEM or autoimmune encephalopathies were considered. Repeat CSF analysis showed no pleocytosis, normal glucose and protein. Antibody screening including serum and CSF autoimmune encephalopathy panels, serum anti-aquaporin-4 IgG and myelin oligodendrocyte glycoprotein antibodies were negative. Malignancy and infection were considered unlikely given improvement of some of the previously seen lesions without treatment. 
Clinically, she improved after a few days and was discharged home. Subsequently, she had two additional hospitalizations with similar presentations. Given the recurrent episodes without a clear diagnosis, she underwent biopsy of brain and meninges near a left frontal lesion, which revealed thick and hyalinized leptomeningeal and cortical vessel walls strongly positive for ßamyloid by immunohistochemical staining (Figure 2). Additionally, subcortical vessels showed widened perivascular spaces and sparse perivascular inflammatory cells.

Based on her clinical presentation, imaging and biopsy results, she was diagnosed with cerebral amyloid angiopathy-related inflammation (CAA-ri).

\section{Section 4}

\section{What treatment would you consider?}

She received intravenous solumedrol $1 \mathrm{~g}$ daily for 5 days followed by prednisone $60 \mathrm{mg}$ daily, tapered to discontinuation over 12 weeks. She was started on mycophenolate $1000 \mathrm{mg}$ twice daily and continued on levetiracetam $2000 \mathrm{mg}$ and lacosamide $200 \mathrm{mg}$, both twice daily.

She returned to baseline, except for subjective word finding difficulties, and remained seizurefree. A brain MRI 3 months after starting treatment showed marked improvement (eFigure2).

\section{Discussion}

CAA is a disorder seen in the elderly, characterized by deposition of amyloid- $ß$ in small and medium-sized cortical and leptomeningeal arteries. Patients present with spontaneous symptomatic lobar hemorrhage, cognitive impairment, and transient focal neurological episodes. ${ }^{9,10}$ The imaging features include lobar microbleeds, cortical superficial siderosis (cSS), WMH, cortical microinfarcts, cortical atrophy, and centrum semiovale MRI-visible perivascular spaces. $^{9}$ 
Less commonly, CAA can present as a spectrum of inflammatory responses to the deposition of amyloid- $ß$ in the vessel wall. The inflammatory forms include CAA-ri, with perivascular inflammation, and amyloid- $ß$ related angiitis (ABRA), with transmural inflammation of the vessel wall. ${ }^{11-14}$ The pathophysiology of CAA is thought to be related to protein elimination failure in the perivascular drainage pathways, with amyloid-ß deposits along the basement membrane ${ }^{10}$ triggering an immune response. ${ }^{12,13}$

CAA-ri occurs in younger individuals than those with conventional CAA, with a mean age of onset of $67 .{ }^{13}$ Women and men are affected equally. CAA-ri manifests with acute or subacute cognitive and behavioral changes, headache, focal neurological deficits, or seizures. ${ }^{12,13} \mathrm{MRI}$ reveals $\mathrm{WMH}$, predominantly posterior, typically asymmetric and extending to the immediately subcortical WM, along with associated edema. ${ }^{10,13}$ Microhemorrhages are frequently present, often of maximal concentration in areas of abnormal WM and in lobar locations, in contrast to the deep location seen in patients with hypertensive arteriopathy. ${ }^{15}$ At times, leptomeningeal or parenchymal enhancement is seen. ${ }^{13}$ Another radiological feature is enlarged perivascular spaces, due to impairment of perivascular drainage by amyloid- $§$ deposition, ${ }^{16}$ seen in the centrum semiovale, as opposed to the basal ganglia in patients with hypertensive arteriopathy. ${ }^{15}$ Patients can have increased inflammatory markers in serum, and mild to moderate pleocytosis and elevated protein in the CSF. ${ }^{13}$

A definite diagnosis requires histopathological confirmation; however a diagnosis of possible or probable CAA-ri is made using diagnostic criteria ${ }^{14}$ (eTable) based on clinical ( $\geq 40$-years-old, presence of $\geq 1$ clinical features: headache, impaired consciousness, behavioral changes, focal neurological signs, or seizures) and radiological findings (asymmetric WMH involving U-fibers, and $\geq 1$ cerebral macro or microbleeds or cSS), and the exclusion of other causes. These criteria have sensitivity and specificity of $82 \%$ and $97 \%$, respectively, for probable CAA-ri, and $82 \%$ and $68 \%$, respectively, for possible CAA-ri. ${ }^{14}$ Stereotactic brain biopsy is generally safe; however, it is important to balance the risks of brain biopsy versus empirical immunosuppression. Considering biopsy only after lack of clinico-radiological improvement after 3 weeks of immunosuppressants has been proposed. ${ }^{13}$ 
A retrospective study ${ }^{17}$ found that immunosuppression, with varied regimens including corticosteroids, cyclophosphamide, and mycophenolate, improves the clinical, radiographic, and recurrence rates in patients with CAA-ri.

Our patient's clinical features, including headache, focal neurological deficits and seizures, and radiological findings of multifocal $\mathrm{WMH}$, microhemorrhages, and enlarged perivascular spaces, supported a diagnosis of probable CAA-ri. Although histological perivascular inflammatory changes were not prominent, this could be attributed to sampling due to the focal and segmental nature of the inflammatory changes. ${ }^{11}$ The significant clinical and radiographical improvement after initiation of immunosuppression further supports the diagnosis.

Albeit uncommon, CAA-ri is an important diagnosis to consider in the proper clinical setting because most patients respond to immunosuppressive therapy. 


\section{Figure legends}

Figure 1. (A) Fluid-attenuated inversion recovery (FLAIR) demonstrates multiple cortical and subcortical white matter FLAIR hyperintense signal changes that involve the subcortical U fibers, most prominently within the right frontoparietal, left temporo-occipital, and left greater than right occipital lobes. (B) Diffusion-weighted imaging (DWI, on the left) and apparent diffusion coefficient sequences ( $A D C$, on the right) show cortical diffusion restriction and vasogenic edema, respectively, in the left temporo-occipital region. (C) Gradient echo sequence (GRE) reveals punctate microhemorrhages in the right parietal lobe.

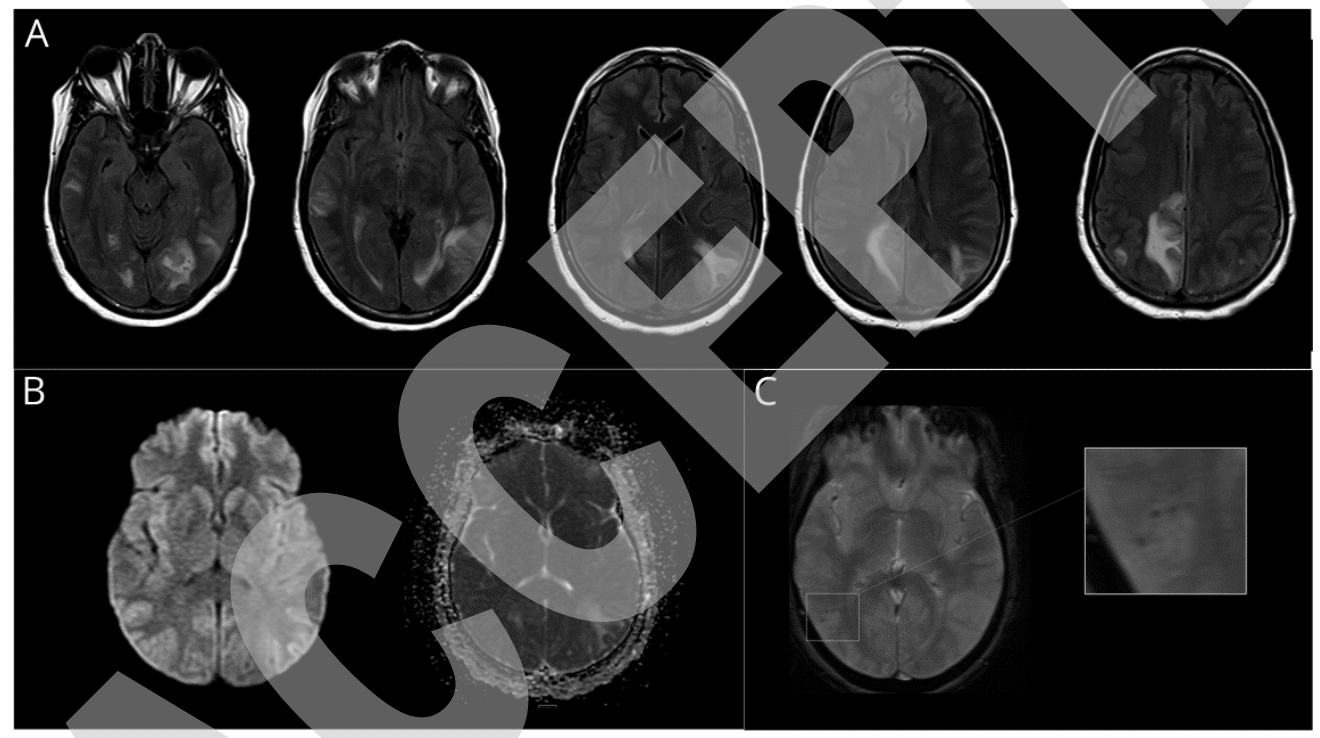


Figure 2. Biopsy of the leptomeninges, cortex, and subcortical white matter showed (A) small arteries with thickened and hyalinized walls on hematoxylin and eosin stain, 400x (see arrows), containing eosinophilic material that is positive for ß-amyloid by immunohistochemical staining (B), 200x.
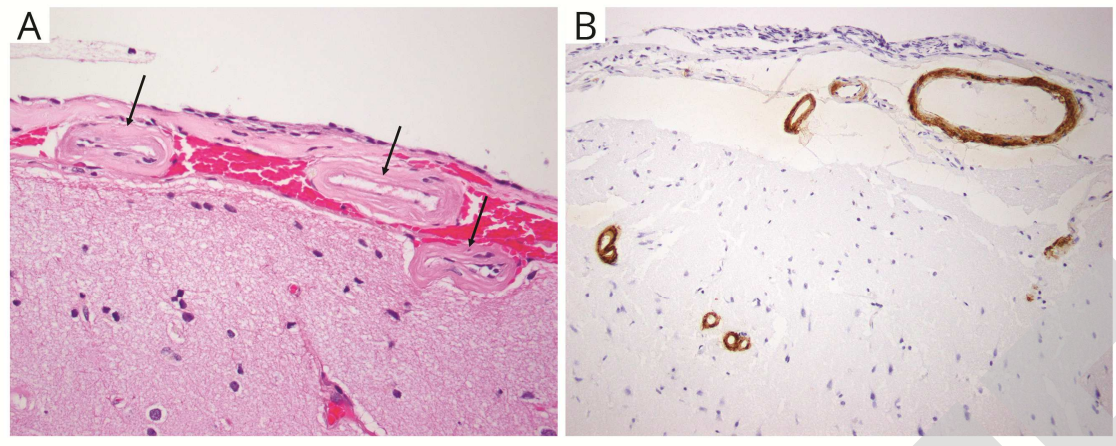

\section{Supplement -- http://links.lww.com/WNL/B709}

\section{References}

1. Bender MB, Feldman M, Sobin AJ. Palinopsia. Brain. Jun 1968;91(2):321-38. doi:10.1093/brain/91.2.321

2. Rossetti Y, Pisella L, McIntosh RD. Definition: Optic ataxia. Cortex. Dec 2019;121:481. doi:10.1016/j.cortex.2019.09.004

3. Fugate JE, Rabinstein AA. Posterior reversible encephalopathy syndrome: clinical and radiological manifestations, pathophysiology, and outstanding questions. Lancet Neurol. Sep 2015;14(9):914-925. doi:10.1016/s1474-4422(15)00111-8

4. Cianfoni A, Caulo M, Cerase A, et al. Seizure-induced brain lesions: a wide spectrum of variably reversible MRI abnormalities. Eur J Radiol. Nov 2013;82(11):1964-72. doi:10.1016/j.ejrad.2013.05.020

5. Godi C, De Vita E, Tombetti E, Davagnanam I, Haddow L, Jäger HR. High b-value diffusion-weighted imaging in progressive multifocal leukoencephalopathy in HIV patients. Eur Radiol. Sep 2017;27(9):3593-3599. doi:10.1007/s00330-017-4761-8

6. Soun JE, Song JW, Romero JM, Schaefer PW. Central Nervous System Vasculopathies. Radiol Clin North Am. Nov 2019;57(6):1117-1131. doi:10.1016/j.rcl.2019.07.005

7. Cappelen-Smith C, Calic Z, Cordato D. Reversible Cerebral Vasoconstriction Syndrome: Recognition and Treatment. Curr Treat Options Neurol. Jun 2017;19(6):21. doi:10.1007/s11940017-0460-7

8. Pilato F, Distefano M, Calandrelli R. Posterior Reversible Encephalopathy Syndrome and Reversible Cerebral Vasoconstriction Syndrome: Clinical and Radiological Considerations. Front Neurol. 2020;11:34. doi:10.3389/fneur.2020.00034 
9. Wermer MJH, Greenberg SM. The growing clinical spectrum of cerebral amyloid angiopathy. Curr Opin Neurol. Feb 2018;31(1):28-35. doi:10.1097/wco.0000000000000510

10. Charidimou A, Boulouis G, Gurol ME, et al. Emerging concepts in sporadic cerebral amyloid angiopathy. Brain. Jul 1 2017;140(7):1829-1850. doi:10.1093/brain/awx047

11. Scolding NJ, Joseph F, Kirby PA, et al. Abeta-related angiitis: primary angiitis of the central nervous system associated with cerebral amyloid angiopathy. Brain. Mar 2005;128(Pt 3):500-15. doi:10.1093/brain/awh379

12. Eng JA, Frosch MP, Choi K, Rebeck GW, Greenberg SM. Clinical manifestations of cerebral amyloid angiopathy-related inflammation. Ann Neurol. Feb 2004;55(2):250-6. doi:10.1002/ana.10810

13. Chung KK, Anderson NE, Hutchinson D, Synek B, Barber PA. Cerebral amyloid angiopathy related inflammation: three case reports and a review. Journal of neurology, neurosurgery, and psychiatry. Jan 2011;82(1):20-6. doi:10.1136/jnnp.2009.204180

14. Auriel E, Charidimou A, Gurol ME, et al. Validation of Clinicoradiological Criteria for the Diagnosis of Cerebral Amyloid Angiopathy-Related Inflammation. JAMA Neurol. Feb 2016;73(2):197-202. doi:10.1001/jamaneurol.2015.4078

15. Charidimou A, Boulouis G, Pasi M, et al. MRI-visible perivascular spaces in cerebral amyloid angiopathy and hypertensive arteriopathy. Neurology. Mar 21 2017;88(12):1157-1164. doi:10.1212/wnl.0000000000003746

16. Charidimou A, Gang Q, Werring DJ. Sporadic cerebral amyloid angiopathy revisited: recent insights into pathophysiology and clinical spectrum. Journal of Neurology, Neurosurgery and Psychiatry. 2012;83(2):124. doi:10.1136/jnnp-2011-301308

17. Regenhardt RW, Thon JM, Das AS, et al. Association Between Immunosuppressive Treatment and Outcomes of Cerebral Amyloid Angiopathy-Related Inflammation. JAMA Neurol. Jun 22 2020;77(10):1-10. doi:10.1001/jamaneurol.2020.1782

Appendix 1. Authors.

\begin{tabular}{|l|l|l|}
\hline Name & Location & Contribution \\
\hline Valerie Jeanneret, MD & Emory University, Atlanta & $\begin{array}{l}\text { Literature review and } \\
\text { manuscript drafting }\end{array}$ \\
\hline Stewart Neil & Emory University, Atlanta & $\begin{array}{l}\text { Literature and manuscript } \\
\text { review }\end{array}$ \\
\hline Greene, James G & Emory University, Atlanta & $\begin{array}{l}\text { Literature and manuscript } \\
\text { review }\end{array}$ \\
\hline Olivia Groover & Emory University, Atlanta & $\begin{array}{l}\text { Literature and manuscript } \\
\text { review }\end{array}$ \\
\hline
\end{tabular}




\begin{tabular}{|l|l|l|}
\hline Carlos S Kase, MD & Emory University, Atlanta & $\begin{array}{l}\text { Literature and manuscript } \\
\text { review }\end{array}$ \\
\hline
\end{tabular}




\title{
Neurology
}

\author{
Clinical Reasoning: A 55-Year-Old Woman With Recurrent Episodes of Aphasia and \\ Vision Changes \\ Valerie Jeanneret, Stewart Neill, James G Greene, et al. \\ Neurology published online December 14, 2021 \\ DOI 10.1212/WNL.0000000000013219
}

This information is current as of December 14, 2021

\section{Updated Information \&} Services

Subspecialty Collections

Permissions \& Licensing

Reprints including high resolution figures, can be found at: http://n.neurology.org/content/early/2021/12/13/WNL.0000000000013219.f ull

This article, along with others on similar topics, appears in the following collection(s):

All Clinical Neurology

http://n.neurology.org/cgi/collection/all_clinical_neurology Autoimmune diseases

http://n.neurology.org/cgi/collection/autoimmune_diseases

Intracerebral hemorrhage

http://n.neurology.org/cgi/collection/intracerebral_hemorrhage

Information about reproducing this article in parts (figures,tables) or in its entirety can be found online at:

http://www.neurology.org/about/about_the_journal\#permissions

Information about ordering reprints can be found online:

http://n.neurology.org/subscribers/advertise

Neurology $\AA^{\circledR}$ is the official journal of the American Academy of Neurology. Published continuously since 1951, it is now a weekly with 48 issues per year. Copyright (C) 2021 American Academy of Neurology. All rights reserved. Print ISSN: 0028-3878. Online ISSN: 1526-632X.

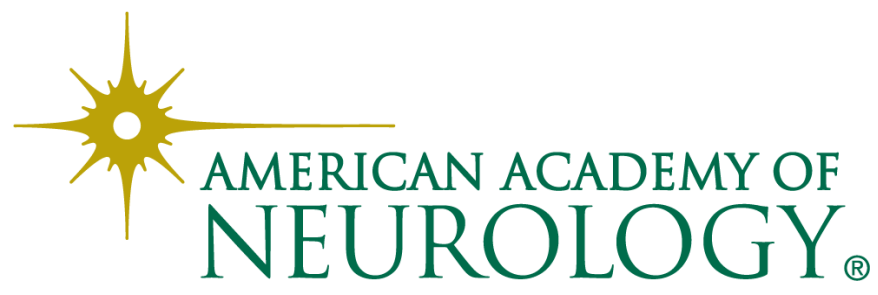

